\title{
THE EFFECT OF SELECTIVE, CHRONIC STIMULATION ON MOTOR UNIT SIZE IN DEVELOPING RAT MUSCLE ${ }^{1}$
}

\author{
R. M. A. P. RIDGE AND W. J. BETZ ${ }^{2}$ \\ Department of Physiology, University of Bristol, University Walk, Bristol BS8 1TD, England
}

Received January 24, 1984; Revised April 16, 1984; Accepted April 17, 1984

\begin{abstract}
One of the two peripheral nerves which innervate rat lumbrical muscle was stimulated chronically in vivo during the postnatal period of synapse elimination to determine whether the differential stimulation would affect the outcome of the elimination process. Rats were anesthetized for about $4 \mathrm{hr}$ a day for 5 to 6 consecutive days, during which time the sural nerve (or, in other animals, the lateral plantar nerve) was electrically stimulated. Each animal received about $10^{6}$ stimuli. After the last stimulation period, the sizes of motor units in both nerves were estimated from motor unit tension recorded in vitro. We found that, on average, sural motor units were larger than others in animals which had received sural nerve stimulation and smaller than others in animals which had received lateral plantar nerve stimulation. These results arc consistent with the hypothesis that more active nerve terminals possess a relative advantage in competing for occupancy of the endplate.
\end{abstract}

The postnatal development of mammalian muscle involves a series of changes guided by interactions between muscle fibers and motor nerves. Previously we have studied some of these changes in rat lumbrical muscle, using electrophysiological and histological techniques. At birth, about two-thirds of the adult number of muscle fibers and all of the motor axons are present in the lumbrical muscle, and each muscle fiber is innervated by two or three separate motor axons (Betz et al., 1979). Over a period of about 3 weeks, new muscle fibers appear, and virtually all muscle fibers become innervated by a single motor axon. The appearance of new muscle fibers depends on the presence of the motor axons (since denervation arrests new muscle fiber production), and the elimination of all but one synaptic input to each muscle fiber is based on competition between converging axons, since no discernible synapse elimination occurs in muscles in which all but one motor axon are cut (Betz et al., 1980a).

It has been suggested that the competition between converging synapses depends somehow on nerve and/or muscle activity. For instance, blocking action potentials in a nerve slows the rate of elimination (Thompson et al., 1979), and electrical stimulation of muscle and nerve increases the rate of elimination (O'Brien et al., 1978; Thompson, 1983). These experiments involved uniform changes in the activity of all motor axons to a muscle. It is not possible to predict from these experiments

\footnotetext{
${ }^{1}$ We are grateful to Dr. R. R. Ribchester for sharing unpublished data, for helpful discussions, and for his assistance with the manuscript, and to Dr. J. H. Caldwell for advice and comments on the manuscript. Travel support for W. J. B. was provided by the Muscular Dystrophy Association of America.

${ }^{2}$ To whom correspondence should be directed, at his permanent address: Department of Physiology, University of Colorado School of Medicine, Denver, CO 80262.
}

whether nonuniform activity would confer a relative advantage or disadvantage, or have no effect, on synapse elimination by more active, compared to less active, axons. That is, while the rate of elimination is clearly activity dependent, the outcome of the competition (i.e., which axon wins) may or may not be activity dependent.

Recently, Ribchester and Taxt (1983) have shown that the amount of activity does affect the competition between converging axons in adult rat lumbrical muscles undergoing reinnervation. They found that following nerve crush, if part of the nerve supply to the muscle is chronically blocked by tetrodotoxin (TTX), the blocked axons reinnervate a smaller number of muscle fibers than normal, while axons not blocked by TTX reinnervate a larger number of muscle fibers than normal. This suggests that active axons compete more effectively than blocked axons for synaptic sites in adults.

We wondered whether a similar mechanism could operate during the elimination of synapses in neonatal animals. In the present experiments, we chronically stimulated one of the two nerves which innervate the lumbrical muscle, and then we measured individual motor unit tensions as a way of estimating motor unit size. One of the nerves (the sural nerve, SN) usually supplies 1 or 2 motor axons to the muscle, while the other (the lateral plantar nerve, LPN) supplies 8 to 10 axons. If more active motor units have a competitive advantage, then sural motor unit size should be relatively larger in animals receiving SN stimulation and relatively smaller in animals receiving LPN stimulation. While our sample size was small (8 of 50 animals completed the protocol), the results were consistent with these predictions.

\section{Materials and Methods}

The rat lumbrical muscle receives its innervation from two separate peripheral nerves. Most (8 to 10) motor axons are found in the LPN, 
with an additional 1 or 2 axons usually supplied by the SN. In the lower leg, the two nerves are widely separated (they join near the heel), and so their electrical activity can be independently controlled in vivo by blocking agents (Betz et al., 1980b; Ribchester and Taxt, 1983) or by electrical stimulation (see below).

Deafferentation. All animals in the study were unilaterally deafferented in order to abolish flexion withdrawal reflexes and reflex activation of other motor neurons during nerve stimulation. 'I'his procedure causes no significant change in the time course of synapse elimination in the lumbrical muscle (Caldwell and Ridge, 1983).

Fifty animals were deafferented 6 (or occasionally 7 ) days after birth. The animals were anesthetized with ether. A laminectomy was performed, and the dorsal roots were cut intradurally and central to the dorsal root ganglia, on the right side, in spinal cord segments L2 to S2. The exposed surface of the cord was then covered with a thin layer of gelatin sponge (Sterispon no. 2, Allen and Hanbury, Ltd.), and the skin was sutured. The effectiveness of the operation was tested after recovery from anesthesia by gently pinching the skin of the leg and foot with forceps and noting the animal's response (cf. Caldwell and Ridge, 1983).

Nerve stimulation. Beginning the day after deafferentation, either the SN or LPN in one leg was stimulated for about $4 \mathrm{hr} /$ day for 5 to 6 days.

The animals were anesthetized with sodium pentobarbitone (Sagatal, May \& Baker, Ltd.) injected intraperitoneally. The first injection was at 20 to $40 \mathrm{mg} / \mathrm{kg}$, the solution supplied $(60 \mathrm{mg}$ in $1 \mathrm{ml})$ being diluted $1: 5$ with $0.9 \% \mathrm{NaCl}$ solution. Subsequent doses (applied usually at hourly intervals, when spontaneous movements appeared) were at 10 to $15 \mathrm{mg} / \mathrm{kg}$, the stock solution being diluted $1: 10$. We aimed for light anesthesia. As the animals were deafferented, electrical stimulation caused no pain. The animals were lightly restrained with a tape across the back and another across the upper foot. They were kept warm with a lamp and a small blanket, the temperature being monitored with a thermometer under the blanket $\left(28\right.$ to $\left.30^{\circ} \mathrm{C}\right)$.

Usually nerves were stimulated in four animals simultaneously at four separate stations along a laboratory bench, each equipped with a stimulator and dissection microscope. The nerve was surgically exposed in the lower leg; the SN lies on the posterior aspect and the LPN on the medial aspect of the leg. Stimuli $(10-\mu$ sec pulses, usually about 10 $\mathrm{V}, 40 / \mathrm{sec}$ for $2 \mathrm{sec}$ every $5 \mathrm{sec}$ ) were delivered via cotton wick electrodes. These consisted of two glass pipettes filled with $0.9 \%$ saline with cotton threaded through the tips (extending 3 to $6 \mathrm{~mm}$ ). Inside the pipette a chlorided silver wire was tightly wound around the cotton thread.

Each animal was observed through a dissection microscope every 2 to $10 \mathrm{~min}$ during the stimulation period to assess the effectiveness of the stimulation. Contraction of the fourth deep lumbrical muscle (which was the muscle used in acute experiments; see below) causes flexion of the fifth digit. Observed flexion of this digit usually provided a fairly reliable index of adequate stimulation of the nerve (especially when the LPN was stimulated, since it provides most of the innervation to the muscle). In the youngest animals, unavoidable direct stimulation of muscles in the lower leg caused foot and some toe flexion, but this could usually be distinguished from nerve-evoked contraction of the fourth lumbrical.

Of the 50 animals deafferented, 8 completed the entire experimental protocol, each receiving about $10^{6}$ stimuli, and only results from these 8 are given here. Of the others, 8 were not used (these served as controls for the effects of the deafferentation; all gained weight normally), 21 suffered apparent anesthetic overdose (most of these did not die during stimulation but failed to recover from the anesthesia, even though they were judged to be only lightly anesthetized during the stimulation period), 6 failed to gain weight adequately, and 7 suffered nerve damage during stimulation (recognized by loss of toe flexion during stimulation).

Electrophysiology. One day after the last stimulation period the acute experiment was performed. Fourth lumbrical muscles on stimulated and control sides were dissected, along with the attached LPN and SN. Tension was measured in response to nerve and muscle stimulation.

We used contralateral (unstimulated) muscles as controls. We assume that the antidromic action potentials evoked in the motor axons by the stimulation did not significantly affect the innervation of the contralateral muscle.

The acute methods were essentially the same as those described by Betz et al. (1979). The preparation was mounted in a Sylgard-lined dish and superfused with Ringer's solution of the following composition (millimolar concentration): $\mathrm{Na}^{+}, 149 ; \mathrm{K}^{+}, 5 ; \mathrm{Mg}^{2+}, 1 ; \mathrm{Ca}^{2+}, 2 ; \mathrm{Cl}^{-}, 147$; $\mathrm{HCO}_{3}^{-}, 12 ; \mathrm{H}_{2} \mathrm{PO}_{4}^{-}, 1 ; \mathrm{D}$-glucose, 11. The solution was equilibrated by bubbling with $95 \% \mathrm{O}_{2} / 5 \% \mathrm{CO}_{2}$, and a few drops of phenol red were added per liter of Ringer's solution to monitor $\mathrm{pH}$. The bath tempera ture was kept constant at $25^{\circ} \mathrm{C}$.

Tension was recorded via a silicon strain gauge (Akers AE802), the muscle tendon being attached via a fine pin. The initial muscle length was adjusted for maximum twitch tension development. Strain gauge resonant frequency was about $300 \mathrm{~Hz}$, compliance $60 \mu \mathrm{m} /$ millinewton $(\mathrm{mN})$, and output sensitivity $7.7 \mathrm{mV} / \mathrm{mN}$, being linear well beyond the maximal muscle tensions encountered.

Permanent records were made by photographing the oscilloscope display. The two nerves were stimulated via suction electrodes, stimuli being less than $10 \mathrm{~V}$ and 50 to $100 \mu \mathrm{sec}$ in duration. We measured twitch and tetanic tensions in response to whole nerve stimulation and to direct muscle stimulation. For direct stimulation, the tip of the suction pipette was applied directly to the muscle, and pulses of $100 \mathrm{~V}$ and $1 \mathrm{msec}$ were applied. Single motor unit twitch tensions were measured by grading nerve stimulus strength, changing stimulus polarity and duration, and repositioning the nerve in the electrode. We always isolated every SN motor unit (zero to three, usually one or two units). Usually, two or three units could be isolated from the LPN. We could use only units activated singly because of overlap of motor units due to polyneuronal innervation of muscle fibers. The overlap was not so great as to prevent us from measuring the number of lateral plantar units (usually six to eight). Finally, the nerves were stimulated tetanically, both separately and together.

Histology. After the acute experiment, muscles were fixed in phosphate-buffered formalin. Transverse sections $4 \mu \mathrm{m}$ thick were cut in the midbelly region. Photographs of these sections were used to measure muscle fiber cross-sectional areas with a Hewlett-Packard $9816 \mathrm{com}$ puter and digitizer. The total number of muscle fibers was also counted from photographs of each muscle.

\section{Results}

Each animal received about $10^{6}$ stimuli, delivered in 2-sec trains (40/sec) every $5 \mathrm{sec}$ for about $4 \mathrm{hr}$ each day, over a 5- to 6-day period (see "Materials and Methods"). Muscles were then studied in acute experiments in vitro. Examples of isometric tension recordings from one animal are shown in Figure 1. They show records obtained in order to assess the number of motor units in LPN $(a)$, the level of polyneuronal innervation (b), and the number and size of the SN motor units present (in this case two units, $c$ and $d$ ). All physiological data were obtained from records like these.
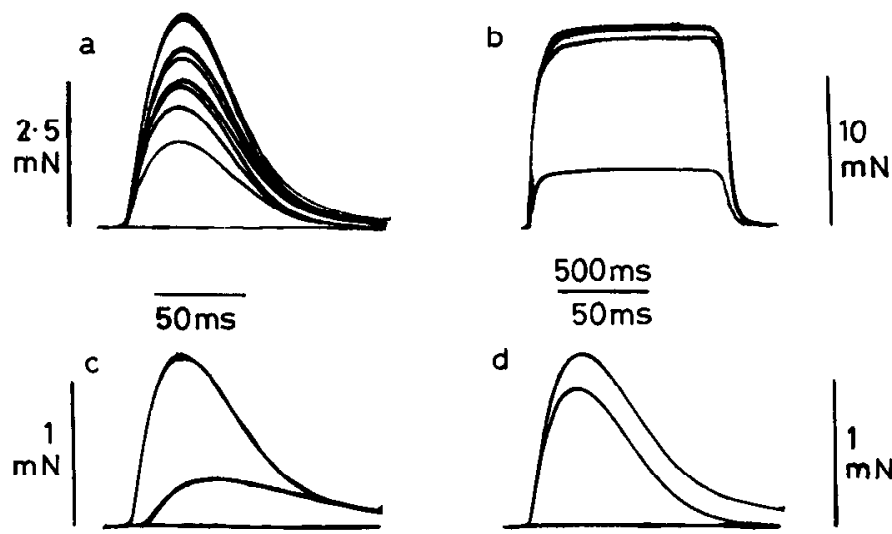

$50 \mathrm{~ms}$

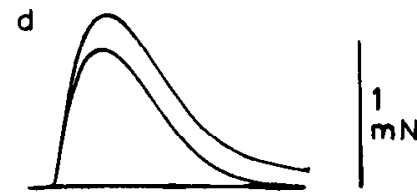

Figure 1. Superimposed traces of tension recordings of isometric twitches and tetani from an animal in which the LPN had been stimulated for 6 days. $a$, Twitches evoked by graded stimulation of the LPN. Eight separate motor units could be counted reliably with repeated trials (top line contains two units as shown by the relaxation phase of the twitch). $b$, Tetanic tension recordings produced by supramaximal stimulation of the SN (lowest trace), LPN (middle trace), or both nerves (top trace). $c$, Twitches evoked by graded stimulation of the SN. Two units can be counted. $d$, Same as $c$ but with the stimulus polarity and order of recruitment of motor units reversed. 


\section{Comparison of motor unit sizes}

Motor unit notation. Throughout the rest of the paper, motor units will be identified by two labels. The first identifies the nerve in which the unit was found (SN or LPN). The second label identifies the type of stimulation received by the muscle innervated by the motor unit (SN stimulation, LPN stimulation, or control (no stimulation)). Thus, in all six types of motor units are distinguished.

Predictions from the hypothesis. If the SN and LPN contained equal numbers of motor units, then the experimental design would be simple. Either one of the nerves would be stimulated, and, if the hypothesis were correct, motor units in the stimulated nerve should become larger at the expense of motor units in the unstimulated nerve. In reality, however, the SN contains at most a few motor axons, whereas the LPN contains 8 to 10 . Thus, stimulating either nerve would have at most a modest effect on the size of individual LPN motor units, since any change would be spread over a relatively large number of LPN motor units. On the other hand, the unequal partitioning of axons in the two nerves should augment the effects of stimulation on SN motor unit size. Thus, the simplest predictions of the hypothesis are that SN units should become larger when the SN is stimulated and smaller when the LPN is stimulated. All other units (SN units/control muscles, LPN units/control muscles, LPN units/LPN stimulated, LPN units/ SN stimulated) should be relatively unaffected.

Further predictions depend on the magnitude of the effect of stimulation. In unstimulated muscles, LPN units recruited are somewhat larger than SN units. This is because in the method of sampling only the first motor unit recruited is measured. Since large motor units tend to be recruited at lower stimulus strengths, and since the LPN contains more units than the SN, the first LPN unit recruited on average will be somewhat larger than the first SN unit (Betz et al., 1979).

A further consequence of the large number of LPN motor units is that one might not expect to observe that LPN units in LPN stimulated muscles are larger than LPN units in control muscles, simply because there is little SN territory to be taken over by LPN units. In addition, in LPN stimulated muscles, increased competition between converging LPN units due to the imposed activity could lead to an increased rate of synapse elimination (O'Brien et al., 1978; Thompson, 1983) with a consequent reduction in LPN unit size in LPN stimulated muscles. Thus, one might predict that LPN units are smaller in LPN stimulated muscles than in control muscles. However, the effect would likely be small, since acute experiments were performed near the end of the normal period of elimination, which would obscure any acceleration of the process due to nerve stimulation.

In summary, the simplest predictions of the hypothesis are that: (1) SN motor units in SN stimulated muscles should be largest, (2) SN units in LPN stimulated muscles should be smallest, and (3) other units should not be altered much by stimulation.

Number of units in the sample. The number of motor units obtained in each category was as follows (number of muscles given in parentheses): $\mathrm{SN}$ units/SN stimulated $=4(4) ; \mathrm{SN}$ units/LPN stimulated $=6(3) ; \mathrm{LPN}$ units/SN stimulated $=11$ (4); LPN units/LPN stimulated $=11(3) ; \mathrm{SN}$ units/control $=$ 13 (7); LPN units/control $=23(8)$.

Hypertrophy of stimulated muscle fibers. If there were significant hypertrophy of the stimulated muscle fibers, this could increase motor unit twitch tension without an increase in the number of muscle fibers in the motor unit. We tested for muscle fiber hypertrophy by measuring a large number of cross-sectional areas of fibers (about half the total in each muscle, selected randomly) in three LPN stimulated muscles. We compared the data with similar measurements from the contralateral controls. The pooled data are shown in Figure 2. The mean cross-sectional areas for control and LPN stimulated muscles are 49.1 and $56.0 \mu \mathrm{m}^{2}$, respectively $(p<0.01)$. Thus, crosssectional area was $14 \%$ greater in LPN stimulated muscles. This is probably a slight underestimate since the sample of

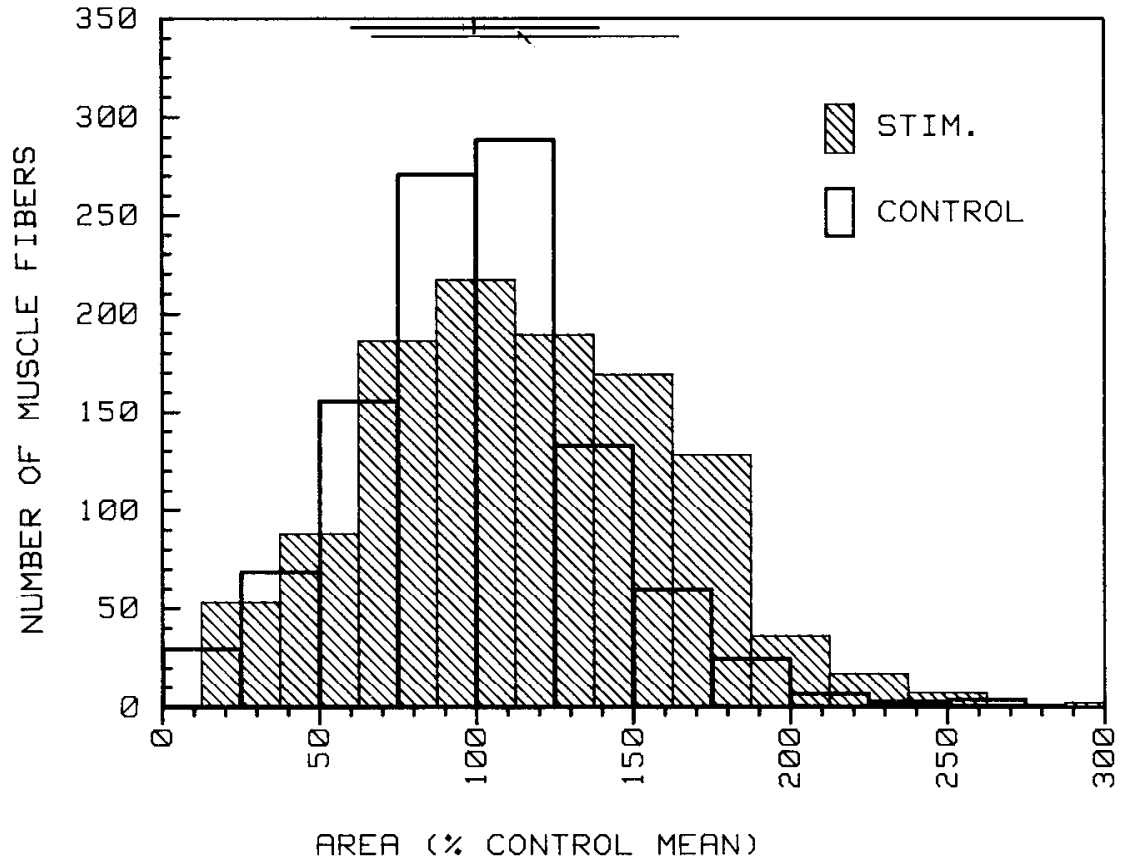

Figure 2. Histograms of muscle fiber cross-sectional areas, expressed for each fiber as percentage of the mean area of all fibers in the control muscle in that animal. Hatched bars, LPN stimulated. Open bars, control, no stimulation. For each type, data were obtained from three muscles; about 500 muscle fibers (randomly selected) were measured in each. Means $\pm 1 \mathrm{SD}$ are shown at the top; the larger mean relates to stimulated muscles. 
LPN stimulated muscles included a small number of fibers which were innervated only by SN units (and, therefore, had not hypertrophied). Offsetting this is the observation that the increase in tension generated in fibers with stimulation-induced hypertrophy is not as great as the increase in cross-sectional area (Lesch et al., 1968; Betz et al., 1980a). Thus, the error due to hypertrophy in motor unit size estimated from tension data is difficult to estimate with certainty, but it probably does not exceed $10 \%$. We have not corrected the data reported here for its effect.

\section{Effect of stimulation on estimates of motor unit size}

Motor unit tension. The average tension (in millinewtons) produced by the six types of motor units is shown in Figure $3 A$ (open symbols). The rank order of the mean values is that predicted by the hypothesis: $\mathrm{SN}$ units in $\mathrm{SN}$ stimulated muscles (open square) were largest, and SN units in LPN stimulated muscles (open triangle) were the smallest. The mean values of the other motor unit types (solid circles) are intermediate; the shaded area was drawn to encompass these values, and the open circle shows the mean value of these units pooled together. All vertical lines show \pm 1 SEM. While the rank order of motor unit tension was predicted by the hypothesis, no comparison was

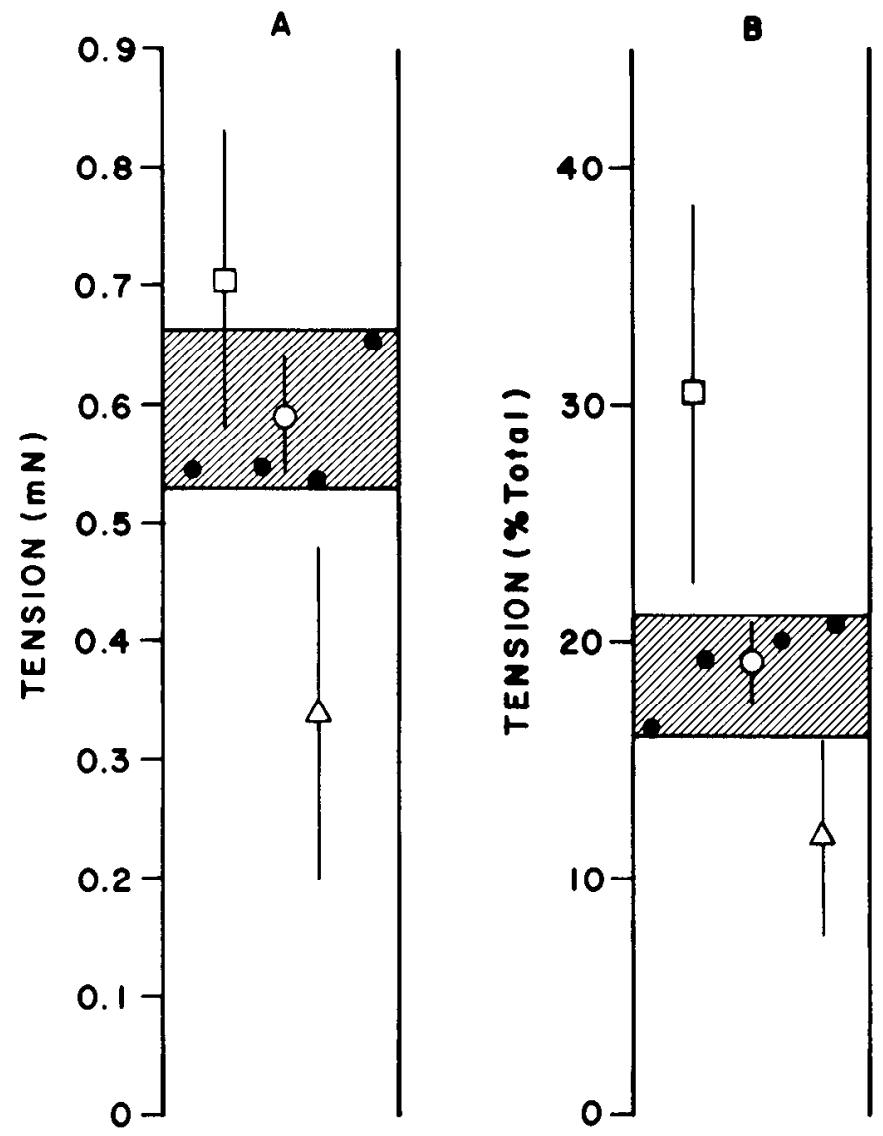

Figure 3. Mean motor unit twitch tensions $(A)$ and normalized twitch tensions $(B)$ of each of the six types of motor units. In both $A$ and $B$, the shaded region encompasses those points (solid circles) for which no significant effect is predicted by the hypothesis (left to right in shaded region (number of units): SN units/control muscles (8), LPN units/LPN stimulated muscles (11), LPN units/SN stimulated (10), and LPN units/control muscles (18)). Pooled mean ( \pm 1 SEM) is shown by the open circle. The hypothesis predicts that $\mathrm{SN}$ units in SN stimulated muscles should be larger (square, $\pm 1 \mathrm{SEM} ; N=4$ ) and that $\mathrm{SN}$ units in LPN stimulated muscles should be smaller (triangle, \pm 1 SEM; $N=6$ ) than normal. None of the differences is statistically significant at the $5 \%$ level (two-tailed $t$ test). statistically significant at the $5 \%$ level, whether using a twotailed $t$ test or the Mann-Whitney rank sum test (Snedecor and Cochran, 1980).

Normalized data. The data shown in Figure $3 A$ are subject to several uncontrolled variables. For instance, animals varied in size and, thus, in the absolute tension generated by motor units. Thus, it was important to normalize the raw data, as was done in Figure $3 B$, where motor unit tensions are expressed as a percentage of the total twitch tension developed when the muscle was directly stimulated. As in Figure $3 A$, the ranking order is that predicted by the hypothesis, although again the differences do not reach the $5 \%$ level of significance, using either a two-tailed $t$ test or rank sum test.

Same animal comparisons. A more comprehensive approach is to compare motor units in the same animal (Fig. 4). For instance, in an animal in which the SN was stimulated, SN units in the stimulated muscle could be compared with each of the other three types of units (LPN units in the same muscle

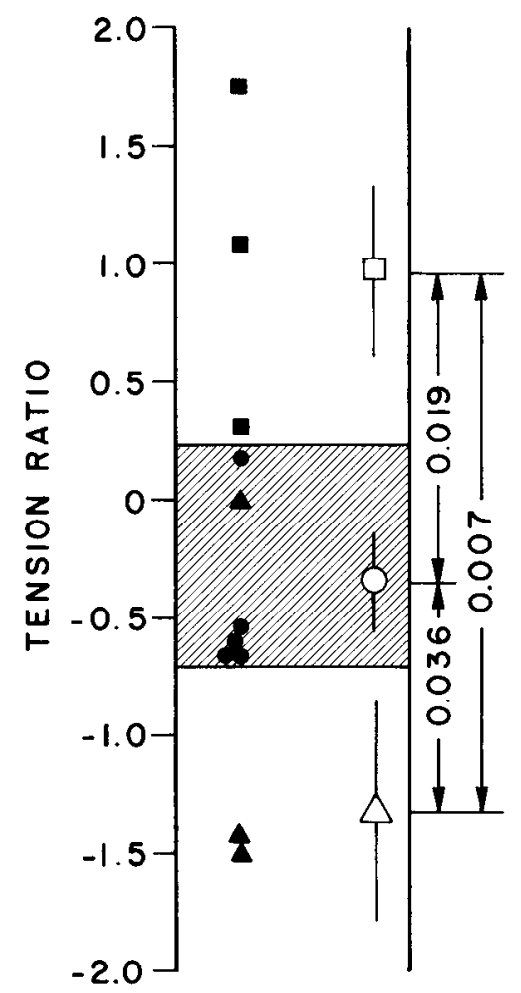

Figure 4. Comparisons between pairs of motor units. Each motor unit was compared with every other motor unit (of different type) in the same animal (see the text for details). Data are represented as in the previous figure. Solid squares show ratios predicted by the hypothesis to be larger than normal (from top down (number of ratios): $\mathrm{SN}$ units/SN stimulated versus $\mathrm{SN}$ units/control muscles (5); $\mathrm{SN}$ units/ SN stimulated versus LPN units/SN stimulated (9); SN units/SN stimulated versus LPN units/control muscles (9)). Pooled mean $( \pm 1$ SEM) is shown by the open square. Solid triangles show ratios predicted to be smaller than normal (from top down $(N)$ : SN units/LPN stimulated versus SN units/control muscles (5); SN units/LPN stimulated versus LPN units/LPN stimulated (21); SN units/LPN stimulated versus LPN units/control muscles (22)). The open triangle shows the pooled mean $( \pm 1$ SEM). Solid circles show ratios for which no effect is predicted. The range covered by these points is shaded. From left to right $(N)$ : SN units/control muscles versus LPN units/SN stimulated (11); SN units/control muscles versus LPN units/LPN stimulated (12); LPN units/SN stimulated versus LPN units/control muscles (21); LPN units/LPN stimulated versus LPN units/control muscles (45); SN units/control muscles versus LPN units/control muscles (24). The open circle shows the pooled mean $( \pm 1$ SEM) of these points. Results $(p$ values) from two-tailed $t$ tests on the pooled data are shown at right. 
and SN and LPN units in the contralateral control muscle). The hypothesis predicts simply that SN units in SN stimulated muscles should be larger than any other units, and, in animals in which an LPN was stimulated, SN units in the LPN stimulated muscle units should be smaller than any of the other units. Thus, with this method each motor unit was compared with every other motor unit of a different type in the same animal.

This procedure generates comparisons between pairs of motor units (of different types) in the same animal and produces a more complete analysis of the effects of stimulation. Since each animal contained four types of units (SN and LPN units on stimulated and control sides), a total of six types of paired comparisons was possible for each animal. There were two kinds of animals (SN stimulation or LPN stimulation), so that a total of 12 different types of comparisons were possible. Finally, comparing control units (SN and LPN units in control muscles) should be the same regardless of which nerve was stimulated; consequently, control comparisons (SN versus LPN units in control muscles) in animals receiving SN stimulation were grouped with control comparisons in animals receiving LPN stimulation, making a total of 11 categories of comparisons. These are the solid symbols in Figure 4.

The following procedure was adopted. For each pair of motor units (of different type, in the same animal), a ratio of twitch tension was calculated by dividing the larger value by the smaller value (thus, the ratio was never less than 1 ). Then 1.0 was subtracted from the ratio (so that units of equal size gave a value of 0.0 ; a value of 1.0 means that units differed by a factor of 2). Next, if the ratio involved comparison of an SN and an LPN unit, the ratio was expressed as positive if $\mathrm{SN}$ was larger and negative if LPN was larger. (If the comparison involved two SN units or two LPN units, the ratio was expressed as positive if the unit on the stimulated side was larger than the unit on the control side and negative if the control unit was larger.)

This procedure has the effect that increases and decreases appear as equal displacements on the graph above and below the zero reference line (which represents equal size motor units). The procedure avoids the hyperbolic effect of simple ratios, in which the displacement of a particular value is different from the displacement of its inverse value.

In Figure 4, as in Figure 3, the shaded area encompasses all comparisons (solid circles) for which the hypothesis predicts no effect. The open circle shows the mean $( \pm 1$ SEM) of data pooled from the solid circles. Similarly, solid squares show mean values of comparisons predicted to be larger than normal by the hypothesis (i.e., SN units in SN stimulated muscles compared to other types of units), with the open square showing the pooled mean \pm 1 SEM of these comparisons. Finally, solid triangles show results predicted to be smaller than normal by the hypothesis (i.e., SN units in LPN stimulated muscles compared to other types of units); the open triangle shows the mean $\pm 1 \mathrm{SEM}$ of data pooled from the solid triangles.

As before, the rank order is closely predicted by the hypothesis; the one variant (the triangle within the shaded area) contained only five comparisons. In addition, the differences are highly significant statistically; results ( $p$ values) of twotailed $t$ tests of differences between the pooled means are shown on the figure; all are considerably less than 0.05. Similar results were obtained using the two-tailed Mann-Whitney rank sum test.

Estimates of polyneuronal innervation. When two or more nerves innervate a muscle, it is possible to estimate the fraction of muscle fibers which receive input from more than one motor neuron (polyneuronal innervation) by measuring the tension produced by stimulating each nerve and by counting the number of motor units in each nerve. The average fraction $f$ of muscle fibers which are innervated by a single motor unit in either nerve (SN or LPN) is:

$$
f=1-(1-t / T)^{n}
$$

where $t=$ tension produced by stimulating the "test" nerve (either SN or LPN), T = tension produced by stimulating both nerves, and $n=$ number of motor units in the "test" nerve.

The fraction of muscle fibers $(p)$ in a whole muscle which receives polyneuronal innervation is:

$$
p=1-(1-f+1 / N)^{N}
$$

where $N=$ sum of the number of motor units in both nerves.

This relation is derived as follows. In an adult, since each muscle fiber is singly innervated, each motor neuron on average innervates a fraction $(F)$ of muscle fibers given by: $F=1 / N$. In the neonate, the analogous fraction $(f)$ is larger, reflecting the polyneuronal innervation present at this stage. The difference $(f-F)$ represents the fraction of muscle fibers receiving "extra" synapses from one motor neuron in the neonate. Assuming random distribution of the "extra" synapses within the neonatal muscle, the fraction of muscle fibers which do not receive "extra" innervation from any of the $N$ motor neurons is: $(1-(f-F))^{N}$. Thus, the fraction of muscle fibers which do receive "extra" innervation from $N$ motor axons is:

$$
1-(1-(f-F))^{N}=1-(1-f+1 / N)^{N}
$$

This is the estimated fraction of muscle fibers receiving polyneuronal innervation (equation 2).

Polyneuronal innervation was estimated in this way for 13 muscles, calculated from SN and LPN tension data and from the measured number of motor units in each nerve. There was considerable scatter in the data, especially in the estimates from $\mathrm{SN}$ stimulation. This probably reflects the wide variation in motor unit size in neonatal animals (cf. Brown et al., 1976; Betz et al., 1979). The mean ( \pm 1 SD) for all animals was $64 \%$ $( \pm 25)$ polyneuronal innervation. This agrees well with earlier estimates of normal 11- to 12-day-old animals, using both intracellular and tension recordings (Fig. 10 in Betz et al., 1979). There was very little difference between different types of muscles; the means for SN stimulated, LPN stimulated, and control muscles all fell between 63 and $66 \%$.

Twitch to tetanus ratios. Figure 5 shows twitch to tetanus ratios for experimental and control muscles. For each point, the ratio on the control side ( $X$ axis) is plotted against the ratio on the experimental side ( $Y$ axis). 'T'he line represents equality on the two sides of the animal. The six different symbols represent tension measurements of different nerves (SN, LPN, or both nerves together) and different experimental conditions (chronic stimulation of SN or LPN). The points scatter evenly around the line of equality, suggesting that chronic stimulation produced no significant effect on the twitch to tetanus ratio. This suggests that there were not large changes in the amount or distribution of synaptic inputs which were subthreshold to a single stimulus but suprathreshold to tetanic stimulation. Such inputs would tend to decrease the ratio. The presence of even smaller inputs, which were subthreshold even to tetanic stimulation (Taxt, 1983), would not have been detected by this procedure.

\section{Discussion}

The hypothesis we were testing is that a raised level of activity during the period of postnatal synapse elimination affects the outcome of the elimination process by conferring a competitive advantage on more active motor units. This advantage would lead to less withdrawal from muscle fibers by axons in the stimulated nerve, such that at the end of the period of stimulation the stimulated axons would innervate on average a larger number of muscle fibers than axons in control muscles. 


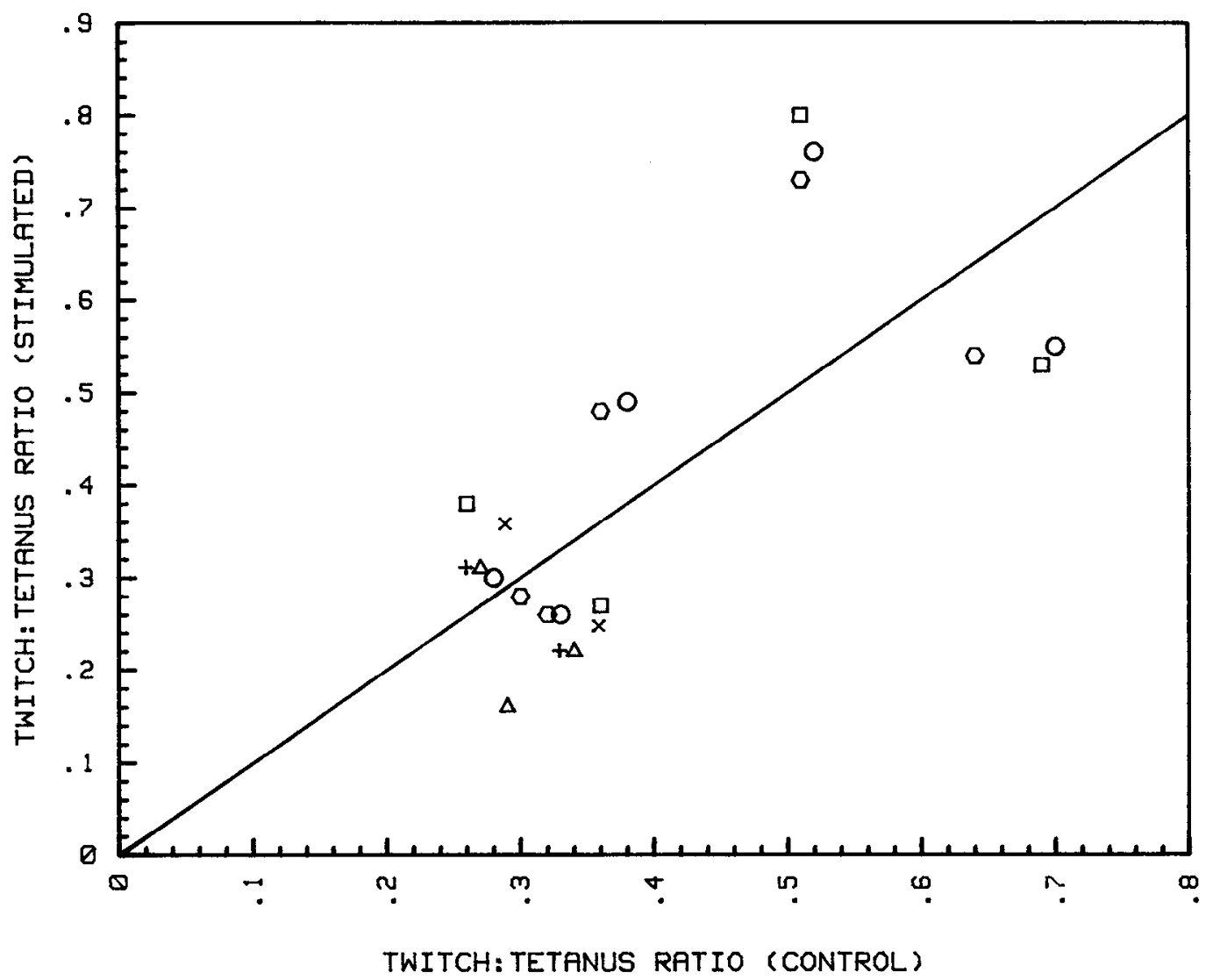

Figure 5. Chronic stimulation did not alter twitch to tension ratios significantly. The twitch to tension ratio measured in acute experiments by stimulating each nerve (SN or LPN) alone or together on the control side ( $X$ axis) is plotted against the ratio obtained by stimulating the homologous nerve on the opposite, experimental side ( $Y$ axis). Each point represents measurements in one animal. The line represents equal twitch to tetanus ratios on the two sides. Squares, SN nerves in chronic SN stimulated animals; triangles, LPN nerves in chronic LPN stimulated animals; hexagons, both nerves in chronic SN stimulated animals; circles, SN nerves in chronic LPN stimulated animals; $X$, LPN nerves in chronic SN stimulated animals; + , both nerves in chronic LPN stimulated animals.

The simplest predictions of this hypothesis are that SN motor units should be relatively large after SN stimulation and relatively small after LPN stimulation. Unfortunately, our sample size was small (especially for stimulated SN motor units), with the result that the simplest tests of the hypothesis, such as a comparison of tensions of different types of motor units (Fig. 3), while showing the predicted rank order, did not show statistically significant differences among the different types of motor units. However, a more comprehensive test, which compared each motor unit with all other units of a different type in the same animal (Fig. 4), showed high levels of statistical significance, in support of the hypothesis. This test produced larger sample sizes than the simple tests in Figure 3 . Thus, it seems reasonable to conclude that the chronic stimulation did produce a small but real effect, as predicted by the hypothesis.

This conclusion does not necessarily mean that activity has only a small effect on synapse elimination. In our experiments stimulation was imposed on otherwise relatively normal activity levels. Larger effects might be observed if activity in the unstimulated nerve were blocked with TTX. Although chronic LPN block with TTX delivered via an implanted osmotic pump has been achieved in adults (Betz et al., 1980b; Ribchester and Taxt, 1983), attempts to block by the same method or by TTXimpregnated cuffs in neonatal rats have failed (W. J. Betz, J. H. Caldwell, and R. R. Ribchester, unpublished observations). Finally, the stimulus regime we used $(40 \mathrm{~Hz}$ for $2 \mathrm{sec}$ followed by $3 \mathrm{sec}$ off, $4 \mathrm{hr}$ /day for 5 to 6 consecutive days) may not be optimal. In adult denervated rat soleus muscle, such a procedure would produce a small but detectable reduction in extrajunctional acetylcholine sensitivity (Lomo and Westgaard, 1975).

Ribchester and Taxt (1983) have recently performed analogous experiments in adult animals undergoing reinnervation. Using the rat lumbrical nerve-muscle preparation, they observed that after the muscle nerve was crushed, axons in the LPN (in which activity was blocked by TTX delivered via an osmotic pump) competed much less successfully with sural motor axons (which were not blocked) for sole occupancy of synaptic sites in the muscle. Our results on neonatal animals are consistent with theirs, suggesting a common mechanism by which activity can influence synapse elimination during development and during reinnervation in the adult. This renders less likely a possible alternative explanation in the studies on adults, namely that results are due to differential effects of activity on sprouting, since signs of sprouting are not present in neonatal animals of the age we used (Betz et al., 1980a).

The dual results reported here- that sural units are relatively larger than normal when the SN is stimulated and relatively smaller than normal when the LPN is stimulated-serve to illustrate the interactive nature of the competitive process underlying synapse elimination. That is, more active nerve fibers not only maintain (and perhaps make new) synaptic contacts with greater tenacity than less active fibers, they apparently also somehow "displace" contacts made by less 
active fibers. The mechanism by which activity produces these effects is unknown; it would be interesting, for instance, to know if it depends upon presynaptic secretion and/or postsynaptic receptor activation.

\section{References}

Betz, W. J., J. H. Caldwell, and R. R. Ribchester (1979) The size of motor units during post-natal development of rat lumbrical muscle. J. Physiol. (Lond.) 297: 463-478.

Betz, W. J., J. H. Caldwell, and R. R. Ribchester (1980a) The effects of partial denervation at birth on the development of muscle fibers and motor units in rat lumbrical muscle. J. Physiol. (Lond.) 303: 256-279.

Betz, W. J., J. H. Caldwell, and R. R. Ribchester (1980b) Sprouting of active nerve terminals in partially inactive muscles of the rat. $\mathrm{J}$. Physiol. (Lond.) 303: 281-297.

Brown, M. C., J. K. S. Jansen, and D. Van Essen (1976). Polyneuronal innervation of skeletal muscle in new-born rats and its elimination during maturation. J. Physiol. (Lond.) 261: 387-422.

Caldwell, J. H., and R. M. A. P. Ridge (1983) The effects of deafferentation and spinal cord transection on synapse elimination in developing rat muscles. J. Physiol. (Lond.) 339: 145-159.
Lesch, M., W. W. Parmley, M. Hamosh, S. Kaufman, and E. J. Sonnenblick (1968) Effects of acute hypertrophy on the contractile properties of skeletal muscle. Am. J. Physiol. 214: 685-690.

Lomo, T., and R. H. Westgaard (1975) Further studies on the control of ACh sensitivity by muscle activity in the rat. $\mathrm{J}$. Physiol. (Lond.) 252: 603-626.

O'Brien, R. A. D., A. J. C. Ostberg, and G. Vrbova (1978). Observations on the elimination of polyneuronal innervation in developing mam malian skeletal muscle. J. Physiol. (Lond.) 282: 571-582.

Ribchester, R. R., and T. Taxt (1983) Motor unit size and synaptic competition in rat lumbrical muscles reinnervated by active and inactive motor axons. J. Physiol. (Lond.) 344: 89-111.

Snedecor, G. W., and W. G. Cochran (1980) Statistical Methods, pp. 144-145, Iowa State University Press, Ames.

Taxt, T. (1983) Local and systemic effects of tetrodotoxin on the formation and elimination of synapses in reinnervated adult rat muscle. J. Physiol. (Lond.) 340: 175-194.

Thompson, W. (1983) Synapse elimination in neonatal rat muscle is sensitive to pattern of muscle use. Nature 303: 614-616.

Thompson, W., D. P. Kuffler, and J. K. S. Jansen (1979) The effect of prolonged, reversible block of nerve impulses on the elimination of polyneuronal innervation of newborn rat skeletal muscle fibers. Neuroscience 4: 271-281. 